\title{
Pediatric inguinal hernia treated by single-port laparoscopic water injection hernia crochet needle
}

\author{
Jian Zhou, Xinxin Chen, Tong Jiang \\ Department of Pediatric Surgery, Wenzhou Women and Children's Hospital, Wenzhou, China
}

Videosurgery Miniinv 2020; 15 (1): 239-244

DOI: https://doi.org/10.5114/wiitm.2019.86799

\begin{abstract}
Introduction: Inguinal hernia is the most common disease in pediatric surgery and is induced by congenital processus vaginalis patency. Almost all inguinal hernias are inguinal indirect hernias, and herniorrhaphy has accounted for about $15 \%$ of all pediatric operations. In the past 20 years, with the development of minimally invasive technology, laparoscopic inguinal herniorrhaphy has been emerging in China and in other countries.

Aim: To introduce a new technique for treating inguinal hernia in children, and evaluate the feasibility and surgical efficacy of a transumbilical single-port laparoscopic water-injection hernia crochet needle in treating pediatric inguinal hernia.

Material and methods: Clinical data of 136 inguinal hernial children treated using a transumbilical single-port laparoscopic water-injection hernia crochet needle in our hospital from June 2017 to December 2018 were retrospectively analyzed.

Results: All procedures were successfully accomplished, and no case was converted to a different procedure; the average operation time was $16 \mathrm{~min}$ for one side and $35 \mathrm{~min}$ for both sides. Patients were followed for an average of 10 months after surgery. One patient had a line-knot reaction at the inguinal puncture point 3 months after surgery and recovered after conservative treatment, and no recurrent case was observed after surgery.

Conclusions: It is feasible to treat pediatric inguinal hernia through a transumbilical single-port laparoscopic water-injection hernia crochet needle, which is associated with safety, less trauma, rapid recovery, no obvious scar, and satisfactory efficacy. Therefore, it is worthy of being promoted and applied in clinical practice.
\end{abstract}

Key words: children, inguinal hernia, single-port laparoscopy, hernia crochet needle.

\section{Introduction}

Inguinal hernia is the most common disease in pediatric surgery and is induced by congenital processus vaginalis patency. Almost all inguinal hernias are inguinal indirect hernias, and herniorrhaphy has accounted for about $15 \%$ of all pediatric operations. With regard to treatment of pediatric inguinal hernia, traditional open surgery mostly makes an abdominal transverse cleavage line incision through the groin, which requires cutting open multiple layers of abdominal wall tissues, separating the spermatic vessels and testicular ducts after finding the hernial sac, and isolating and high ligation of the hernial sac, thereby causing great surgical trauma. In the past 20 years, with the development of minimally invasive technology, laparoscopic inguinal herniorrhaphy has been emerging in China and in other countries [1, 2]. Numerous clinical studies indicate that laparoscopic surgery is obviously superior to traditional surgery for pediatric inguinal hernia, especially for children with bilateral hernias, giant hernia, and recurrent hernia,

\section{Address for correspondence}

JianZhou Joe PhD, Department of Pediatric Surgery, Wenzhou Women and Children's Hospital, Wenzhou, China,

e-mail: zhj20040202@163.com 
which shows a trend towards replacing the traditional open surgery $[3,4]$. Therefore, to confirm the results of previous studies on laparoscopic surgery, this study was conducted to explore the feasibility and surgical efficacy of a transumbilical single-port laparoscopic water-injection hernia crochet needle in treating pediatric inguinal hernia.

\section{Aim}

The objective of this study was to evaluate the feasibility and surgical efficacy of a transumbilical single-port laparoscopic water-injection hernia crochet needle in treating pediatric inguinal hernia.

\section{Material and methods}

\section{Clinical data}

From June 2017 to December 2018, clinical data of 136 children diagnosed through preoperative clinical manifestations and color Doppler ultrasonography, who underwent single-port laparoscopic surgery in our hospital from June 2017 to December 2018, were retrospectively analyzed. Of these patients, 129 were boys and 7 were girls with an average age of 2.3 years (range: 10 months to 11 years). Moreover, 106 cases had a lesion on the right side, 23 on the left side, and 7 on both sides. All children received outpatient postoperative follow-up combined with telephone follow-up for 5-12 months (average: 10 months).

\section{Surgical instrument}

The water-injection hernia crochet needle was provided by Surgaid Medical Device Co., Ltd., Xiamen, China. The puncture part was the double trocar, and the tip was blunt and similar to the epidural puncture ladle needle, which facilitated blunt dissection of the retroperitoneal space from spermatic vessels and testicular ducts; and its tail end could be connected to a $5-\mathrm{ml}$ syringe, which contributed to intraoperative injection of normal saline to separate the retroperitoneal space.

\section{Surgical method}

After successful anesthesia, patients were placed in the supine position, and their hips were appropriately padded. A longitudinal incision of about $5 \mathrm{~mm}$ in length was made through the center of the umbilical area, subcutaneous tissues were bluntly dissect- ed, bilateral abdominal walls were elevated, and the pneumoperitoneum-free needle (5-mm trocar) was directly punctured into the abdominal cavity to generate a pneumoperitoneum pressure of $8-10 \mathrm{~mm} \mathrm{Hg}$. Then, the $30^{\circ}, 5-\mathrm{mm}$ laparoscope was inserted to observe the inner ring opening of the affected side, and conventional exploration of the presence of processus vaginalis patency on the contralateral side was performed to avoid omission.

A 2-mm skin incision was made in the abdominal transverse cleavage line of the affected inguinal region, and the water-injection hernia crochet needle with \#4 silk suture punctured the abdominal muscle layer into the extraperitoneal space in the anterior abdominal wall of the inner ring opening through the skin incision under the laparoscope (Photo $1 \mathrm{~A}$ ). Subsequently, the water-injection hernia crochet needle with silk suture was separated along the medial inner ring opening and passed into the retroperitoneal space to the testicular ducts, and 3-5 ml of normal saline was injected to float and separate the testicular ducts from the retroperitoneum. At this time, the hernia crochet needle had crossed the front of the testicular ducts, punctured the retroperitoneum, and entered the abdominal cavity, while the hernia crochet needle was later slightly withdrawn so that the external silk suture formed a ring. The laparoscopic lens was then pushed forward into that space, the silk suture was introduced, with one end temporarily placed in the abdominal cavity, and the hernia crochet needle was withdrawn along the original path to the extraperitoneal space in the anterior abdominal wall.

The direction of the hernia crochet needle was adjusted and then passed along the lateral inner ring opening to the extraperitoneal space and spermatic vessels. Normal saline was injected continuously, so that the hernia crochet needle could cross the front of the spermatic vessels. Then, the hernia crochet needle was introduced to the abdominal cavity again through the original retroperitoneal puncture site, the needle core was pushed out, the posterior hook slot was used to hook and clamp the preset intraperitoneal silk suture, the preset ligature end was brought out of the body along with the hernia crochet needle, the ipsilateral scrotum was squeezed to evacuate the air, a knot was tied, and the knot was embedded in the extraperitoneal space (Photo $1 \mathrm{~B}$ ).

For children with giant hernia or recurrent hernia in the inguinal region with huge defects, the hernia crochet needle with suture could enter the original 

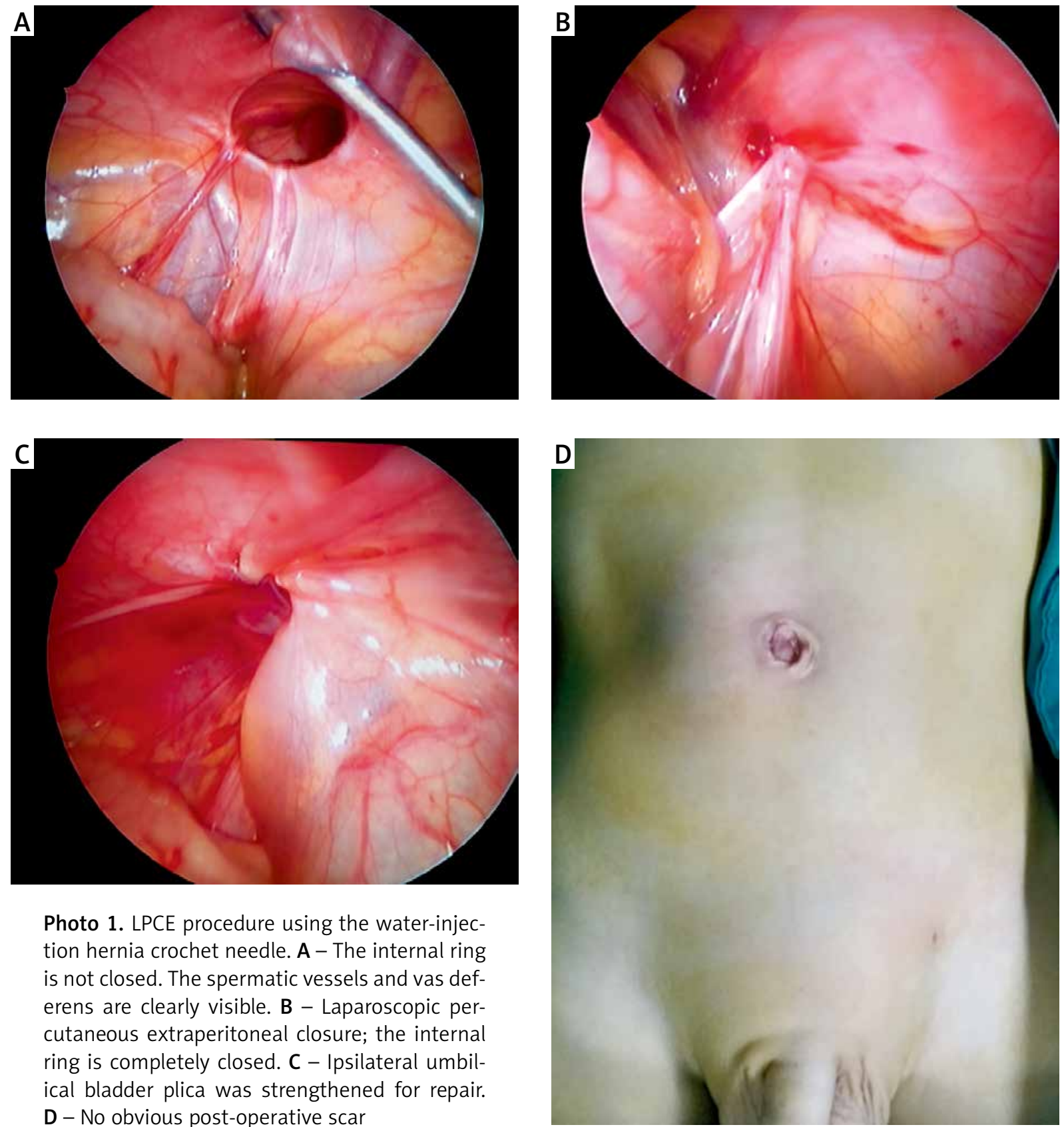

Photo 1. LPCE procedure using the water-injection hernia crochet needle. A - The internal ring is not closed. The spermatic vessels and vas deferens are clearly visible. B - Laparoscopic percutaneous extraperitoneal closure; the internal ring is completely closed. C - Ipsilateral umbilical bladder plica was strengthened for repair. D - No obvious post-operative scar

skin puncture site again after inner ring opening ligation, and the ipsilateral umbilical bladder plica could be punctured using the above surgical method to cover the inner ring opening for enhanced herniorrhaphy (Photo $1 \mathrm{C}$ ). For female patients, the water-injection hernia crochet needle was separated and sneaked into the retroperitoneal space to the round ligament of the uterus, and normal saline was injected to float and separate the round ligament of the uterus from the retroperitoneum. Then, the hernia crochet nee-

dle was passed to the front of the round ligament of the uterus; the remaining surgical procedure was the same as mentioned above. Finally, the umbilical skin was sutured subcutaneously in one stitch, followed by bonding with medical glue to finish the surgery.

\section{Results}

All operations in 136 affected children were successful, and no case was converted to open surgery 
intraoperatively. Of the patients, 35 were diagnosed with unilateral inguinal hernia before surgery, but intraoperative laparoscopic exploration detected the presence of contralateral recessive processus vaginalis patency in 34 boys and 1 girl, and the procedures were completed simultaneously, yielding the incidence of contralateral recessive processus vaginalis patency of $27 \%$ (35/129). The operation times were 13-22 $\mathrm{min}$ for one side (average: $16 \mathrm{~min}$ ) and 28-45 min for both sides (average: $35 \mathrm{~min}$ ). After surgery, the children had mild incisional pain, and no analgesic was needed. Food and off-bed activities were allowed 3-6 $\mathrm{h}$ after surgery, and the patients were discharged 1 day after surgery. The patients were followed for 5-12 months after surgery, and no recurrence was found, but one child developed delayed a line-knot reaction at the inguinal puncture site 3 months after surgery and recovered after conservative treatment.

\section{Discussion}

The main etiology of pediatric inguinal hernia is congenital processus vaginalis patency with increased intra-abdominal pressure induced by factors such as frequent crying and cough. Currently, surgery is the most effective method to cure pediatric inguinal hernia. Traditional open surgery aims to search for the hernial sac by dissecting through the inguinal region and disassociating it to the inner ring opening for high ligation. The hernial sac wall in children is quite thin, and the testicular ducts are fine; thus, excessive disassociation and dissection are likely to result in hernial sac wall avulsion as well as damage to testicular ducts and spermatic vessels. Moreover, it is linked with a high postoperative recurrence rate, as well as a risk of complications such as scrotal hematoma, testicular atrophy, and iatrogenic cryptorchidism $[2,5,6]$.

With the development of minimally invasive medicine and increasing development of various minimally invasive surgical instruments, the pediatric inguinal hernia surgery has gradually transitioned from the traditional open surgery through the groin to minimally invasive laparoscopic surgery and developed from the original three-port and two-port laparoscopy to the latest transumbilical single-port laparoscopic surgery [7-9]. Meanwhile, the closure method of hernial inner ring opening has developed from the initial intraperitoneal suture and ligation to the current laparoscopic percutaneous extraperitoneal closure (LPEC) [10-13]. Moreover, some scholars have also reported the use of various home-made surgical instruments for single-site laparoscopic herniorrhaphy to treat pediatric inguinal hernia, and favorable efficacy has been attained [14]. Compared with the above surgical methods, the water-injection separation technique in single-port laparoscopic surgery used in this study could sufficiently dissociate the spermatic vessels and testicular ducts from the retroperitoneum, which could maximally prevent iatrogenic injury. At the same time, the hernia crochet needle could guarantee that the ligature was entered and withdrawn through the same abdominal wall path, which could avoid ligating the abdominal tissues between the skin and the inner ring opening, thus effectively reducing the recurrence rate after herniorrhaphy. Moreover, it was different from the three-port technique that required specialized skills of laparoscopic operation, as it only required one transabdominal puncture to complete LPEC, without necessitating special training in laparoscopic knotting and suturing. This method was safe, simple, and less invasive, and could be regarded as a minimally invasive surgery in a real sense.

Currently, the treatment for intraoperatively discovered contralateral recessive processus vaginalis patency remains a controversy, and no guideline or expert consensus has suggested that a specific type of recessive processus vaginalis patency requires surgical intervention. In this study, 35 of the 136 affected children were found to have contralateral recessive processus vaginalis patency through laparoscopic exploration, with the incidence of $27 \%$, which was similar to that reported previously (30\%) [15]. A study reported that after unilateral inguinal herniorrhaphy, about $10 \%$ of affected children developed metachronous contralateral inguinal hernia and required a second operation, thus adding to children's suffering and medical expenses [16]. On this account, our experience indicated that laparoscopic macroscopic contralateral recessive processus vaginalis patency should be surgically treated simultaneously.

How to reduce the recurrence rate after single-port laparoscopic inguinal herniorrhaphy in children is a major problem in clinical practice at present, and the most common causes of recurrence include the learning curve factor, indefinite ligation, and ligature loosening $[13,17]$. Research shows that 
although there were individual differences, all trainees acquired the skill to perform LPEC adequately within ten months [18]. Our surgical experience suggested the following points to consider during the surgical operation: (1) After passing the hernia crochet needle through the space between the retroperitoneum and testicular ducts, the needle tip should cling to the adherent testicular ducts, followed by impact injection of normal saline to float the testicular ducts, which favored the smooth passing of the hernia crochet needle through the testicular ducts to avoid damage. (2) When withdrawing the hernia crochet needle for the second time, the crochet needle should be slowly turned to the extraperitoneal space; meanwhile, the ligature should be entered and withdrawn through the same abdominal wall path and should always pass through the same extraperitoneal space. In addition, the ligation should be carried out clinging to the peritoneum to guarantee that no pore is left; the inner ring opening should be completely closed, and attention should be paid to avoid ligating the tissues under abdominal tissues, which may lead to postoperative slipping of the ligature. (3) To avoid a delayed line-knot reaction after surgery, our experience is to slightly elevate the abdominal wall tissues upward upon the completion of ligation and embed the knot under the abdominal muscle layer and outside the anterior wall peritoneum of the inner ring opening, which can remarkably reduce the incidence of postoperative subcutaneous line-knot reaction. (4) For affected children with giant hernia and recurrent hernia, the hernia crochet needle with suture should be used again to puncture the ipsilateral umbilical bladder plica after inner ring opening ligation and make full use of the autologous tissues to cover the inner ring opening to enhance the herniorrhaphy, which can effectively reduce the recurrence rate after surgery. (5) The non-absorbable silk suture should be used as the ligature, while absorbable suture should be used with caution to avoid postoperative recurrence $[19,20]$.

\section{Conclusions}

Numerous novel techniques have emerged in laparoscopic surgery for treating pediatric inguinal hernia, which are developed to increase in vitro knotting and reduce puncture [21, 22]. The results of our clinical cases suggest that transumbilical single-port laparoscopic water-injection hernia crochet needle inguinal herniorrhaphy is a safe and feasible scar-concealing procedure (Photo $1 \mathrm{D})$. With the crochet needle water-injection separation technique, the retroperitoneal space can be more easily separated from the testicular ducts and spermatic vessels. This technique is associated with a simple operation, small surgical injury, high safety, and no obvious body surface scar after surgery. Therefore, this surgical technique can be easily accepted by the parents of affected children and is worthy of being promoted and applied in clinical practice.

\section{Conflict of interest}

The authors declare no conflict of interest.

\section{References}

1. Shehata SM, Attia MA, El Attar AA, et al. Algorithm of laparoscopic technique in pediatric inguinal hernia: results from experience of 10 years. J Laparoendosc Adv Surg Tech A 2018; 28: 755-9.

2. Zhou X, Qi X, Jiang B, et al. Transumbilical endoscopic technique for complete closure of inguinal hernias in female pediatric patients. Exp Ther Med 2017; 13: 41-4.

3. Timberlake MD, Sukhu TA, Herbst KW, et al. Laparoscopic percutaneous inguinal hernia repair in children: review of technique and comparison with open surgery. J Pediatr Urol 2015; 11: 262.e1-6.

4. Raveenthiran V, Agarwal P. Choice of repairing inguinal hernia in children: open versus laparoscopy. Indian J Pediatr 2017; 84: 555-63.

5. Shalaby R, Abd Alrazek M, Elsaied A, et al. Fifteen years experience with laparoscopic inguinal hernia repair in infants and children. J Laparoendosc Adv Surg Tech A 2018; 28: 101-5.

6. Mitura K, Śmietański M, Kozieł S, et al. Factors influencing inguinal hernia symptoms and preoperative evaluation of symptoms by patients: results of a prospective study including 1647 patients. Hernia 2018; 22: 585-91.

7. Korkmaz M, Haluk Güvenç B. Comparison of single-port percutaneous extraperitoneal repair and three-port mini-laparoscopic repair for pediatric inguinal hernia. J Laparoendosc Adv Surg Tech A 2018; 28: 337-42.

8. Xu C, Xiang B, Jin SG, et al. Transumbilical two-port laparoscopic percutaneous extraperitoneal closure: a new technique for inguinal hernia repair in children. J Laparoendosc Adv Surg Tech A 2013; 23: 392-6

9. Ismail M, Shalaby R. Single instrument intracorporeal knot tying during single port laparoscopic hernia repair in children: a new simplified technique. J Pediatr Surg 2014; 49: 1044-8.

10. Becmeur F, Philippe P, Lemandat-Schultz A, et al. A continuous series of 96 laparoscopic inguinal hernia repairs in children by a new technique. Surg Endosc 2004; 18: 1738-41.

11. Emura T, Ohta $\mathrm{H}$, Oyachi $\mathrm{N}$, et al. A modified technique with a new device of laparoscopic percutaneous extraperitoneal 
closure for pediatric inguinal hernia. J Laparoendosc Adv Surg Tech A 2016; 26: 1028-31.

12. Yonggang H, Changfu Q, Ping W, et al. Single-port laparoscopic percutaneous extraperitoneal closure of inguinal hernia using "two-hooked" core needle apparatus in children. Hernia 2019; doi:10.1007/s10029-019-01933-9.

13. Barroso C, Etlinger P, Alves AL, et al. Learing curves for laparoscopic repair of inguinal hernia and communicating hydrocele in children. Front Pediatr 2017; 5: 207.

14. Wang F, Zhong H, Chen Y, et al. Single-site laparoscopic percutaneous extraperitoneal closure of the internal ring using an epidural and spinal needle: excellent results in 1464 children with inguinal hernia/hydrocele. Surg Endosc 2017; 31: 2932-8.

15. Saad S, Mansson J, Sadd A, et al. Ten-year review of groin laparoscopy in 1001 pediatric patients with clinical unilateral inguinal hernia: an improved technique with transhernia multiple-channel scope. J Pediatr Surg 2011; 46: 1011-4.

16. Herbst KW, Thaker H, Lockwood GM, et al. Variation in the use of laparoscopy with inguinal hernia repairs in a sample of pediatric patients at children's hospitals. J Pediatr Urol 2018; 14: 158.e1-7.

17. Xiang B, Jin S, Zhong L, et al. Reasons for recurrence after the laparoscopic repair of indirect inguinal hernia in children. J Laparoendosc Adv Surg Tech A 2015; 25: 681-3.

18. Shibuya S, Fujiwara N, Ochi T, et al. The learning curve of laparoscopic percutaneous extraperitoneal closure (LPEC) for inguinal hernia: protocoled training in a single center for six pediatric surgical trainees. BMC Surg 2019; 19: 6.

19. Grimsby GM, Keays MA, Villanueva C, et al. Non-absorbable sutures are associated with lower recurrence rates in laparoscopic percutaneous inguinal hernia ligation. J Pediatr Urol 2015; 11: 275.e1-4.

20. Kelly KB, Krpata DM, Blatnik JA, et al. Suture choice matters in rabbit model of laparoscopic, preperitoneal, inguinal hernia repair. J Laparoendosc Adv Surg Tech A 2014; 24: 428-31.

21. Li S, Liu L, Li M, et al. Single-port laparoscopic percutaneous extraperitoneal closure using an innovative apparatus for pediatric inguinal hernia. J Laparoendosc Adv Surg Tech A 2014; 24: 188-93.

22. Wang F, Zhong H, Shou T, et al. Single-site laparoscopic percutaneous extraperitoneal closure versus modified transumbilical two-port laparoscopic suturing of the hernia sac for the treatment of pediatric inguinal hernia: comparison of the outcomes of two different approaches. J Laparoendosc Adv Surg Tech A 2019; 29: 103-8.

Received: 27.05.2019, accepted: 23.06.2019. 